\title{
Modelo de estrategias de comprensión lectora para el pensamiento crítico y creativo en estudiantes de educación primaria
}

\author{
Mg. Rosa Cleopatra Gonzaga Contreras \\ rosagc@ucvvirtual.edu.pe \\ https://orcid.org/0000-0002-9256-0974 \\ Modelo Académico de Doctorado en Educación \\ Escuela de postgrado \\ Universidad César Vallejo \\ Chiclayo - Perú
}

\section{RESUMEN}

El estudio respondió a la urgencia de conocer la realidad del pensamiento crítico y creativo, en esa perspectiva, el objetivo general estuvo orientado a proponer un modelo de estrategias de comprensión lectora para el desarrollo de pensamiento crítico y creativo de escolares de segundo grado de educación primaria de la institución educativa 16093, Jaén, 2021. La metodología aplicada correspondió a una investigación de nivel descriptivo-propositiva, usó el método cuantitativo-cualitativo y diseño no experimental. Se utilizó la técnica de la observación para evaluar el pensamiento crítico y creativo, en los 20 estudiantes incluidos en la muestra, instrumento validado a juicio de expertos. Los datos acopiados fueron sometidos a procesamiento con el apoyo de la técnica estadística en su forma descriptiva, además, usando los procesadores SPSS y Excel. Los hallazgos indican que la mayoría de educandos evaluados tienen nivel deficiente en su pensamiento crítico y creativo pues hubo predominancia de este nivel en todas sus dimensiones e indicadores; se concluye que amerita la implementación del modelo de estrategias de comprensión lectora que permitirán revertir la dificultad encontrada.

Palabras clave: modelo de estrategias; comprensión lectora; pensamiento crítico y creativo 


\title{
Reading comprehension strategies model for critical and creative thinking in elementary school students
}

\begin{abstract}
The study responded to the urgency of knowing the reality of critical and creative thinking, in that perspective, the general objective was oriented to propose a model of reading comprehension strategies for the development of critical and creative thinking in second-grade primary school students. of the educational institution 16093, Jaen, 2021. The applied methodology corresponded to a descriptive-purposeful investigation, used the quantitative-qualitative method and non-experimental design. The observation technique was used to evaluate critical and creative thinking in the 20 students included in the sample, an instrument validated by experts. The collected data were subjected to processing with the support of the statistical technique in its descriptive form, in addition, using the SPSS and Excel processors. The findings indicate that the majority of students evaluated have a deficient level in their critical and creative thinking, since there was a predominance of this level in all its dimensions and indicators; It is concluded that it merits the implementation of the reading comprehension strategies model that will make it possible to reverse the difficulty encountered.
\end{abstract}

Keywords: strategy model; reading comprehension; critical and creative thinking

Artículo recibido: 30 noviembre. 2021 Aceptado para publicación: 29 diciembre 2021 Correspondencia: rosagc@ucvvirtual.edu.pe

Conflictos de Interés: Ninguna que declarar 


\section{INTRODUCCIÓN}

En el contexto educativo real se muestra diversas situaciones problemáticas que día a día interfieren en el progreso de aprendizaje de los estudiantes y se ha transformado en una intranquilidad para los educadores, ya que en la labor cotidiana en las aulas de clase y diversos contextos los educandos muestran dificultades diversas que no les permite el fortalecimiento de sus habilidades reflexivas y creativas, las cuales son de suma significación para su progreso personal y social por tanto debería ser el sostén principal de enseñanza de los docentes para formar estudiantes críticos - creativos, autónomos y responsables.

En España, Meneses (2020) en un diálogo sustancioso precisa sobre el objetivo referente a los desafíos de la educación en donde se debe priorizar el futuro de las escuelas, haciendo un análisis reflexivo sobre las diversas leyes educativas que vio nacer y que jamás consiguieron implantar un pacto político y educativo transcendental para afrontar exitosamente las necesidades de los estudiantes, los cuales deben aprender a tener criterios críticos y reflexivos para afrontar las diversas realidades que les toque vivir.

En Latinoamérica, Bretel (2017) Chile, en una entrevista reflexiona sobre la importancia del texto escrito y el saber escribir correctamente ya que esta acción genera el desarrollo de habilidades superiores, el razonamiento y la creatividad, en nuestros estudiantes motivándolos, a desarrollar el hábito de la lectura, análisis y comprensión de textos, activando su razonamiento lógico y su capacidad para crear respuestas y soluciones nuevas, motivando su autonomía.

Por su parte, el informe de la OCDE (2019), establece el gran reto de fomentar la curiosidad, el sentido crítico - reflexivo, la autonomía, la comprensión y el análisis a diferentes situaciones de la vida cotidiana, promoviendo el desarrollo de habilidades superiores para superar dicho reto acorde con la realidad actual. Así mismo, la Unesco (2019) respecto a la educación y su relación con los Objetivos del Desarrollo Sostenible afirma la importancia de la transformación y eficacia en la calidad de educación, su competitividad, la ética de la cooperación, del hacer juntos y de la solidaridad, formando ciudadanos críticos - reflexivos y creativos.

En lo nacional, Torrent (2016) en Lima, manifiesta la importancia del desarrollo del pensamiento crítico y la necesidad de dejar fluir la creatividad y las capacidades naturales de cada uno de los niños y niñas, en donde los maestros deben hacer énfasis en explotar 
su energía y curiosidad de cada uno de ellos fomentando el desarrollo de sus capacidades y destrezas individuales que ellos poseen para así lograr seres humanos críticos - creativos que sean capaces de afrontar diversas situaciones de la vida cotidiana con éxito.

Asimismo, el Ministerio de Educación - MINEDU (2019) realizó un diagnóstico además la conveniencia universitaria de los estudiantes de Pedagogía Básica Regular del Perú, encontrando deficiencias relevantes y aún enfatizó la necesidad de la exposición de las habilidades de altos niveles cognitivos en las cuales se comprometer priorizar la importancia de todos los agentes educativos para poder alcanzar estos objetivos.

En la Región Cajamarca el Proyecto Formativo Departamental (2017) priorizó la asistencia de todos sus actores pedagógicos en la realización de las herramientas de gestión y proposición formativa orientada al acrecentamiento sostenible e implementación del explicación el proyecto censor y creativo implementando el quehacer en redes educativas en el que se plantea una alternativa de misión descentralizada en las áreas rurales de la territorio para entablar el progreso de escuelas innovadoras orientadas al rendimiento de la vigencia educativa garantizando aprendizajes de calidad y perfeccionamiento e inclusión.

Revisando el contexto local, en la Institución Educativa N¹6093 “José Gálvez” de Jaén través de la observación directa a estudiantes del segundo grado de educación primaria y una exploración a docentes de la institución educativa (ver anexo $\mathrm{N}^{\circ} 1$ ) muestran deficiencias en el percepción de habilidades de máxima nivel creativo hecho que se describe mediante su escasa comunicación en las actividades diarias de especie respuestas con fundamento razonable, limitaciones para disponer la colaboración de los personajes de un ejemplar contrariedad para hacer representaciones, deficiencias para abarcar e instituir textos. En las causas se generan el enigma se menciona la destreza pedagógica tradicionales, el de los estudiantes. De no resolverse la situación problemática, los efectos serían, estudiantes pasivos, sin iniciativa, dificultades para ordenar ideas y opiniones, bajos niveles de criticidad y creatividad en la resolución de problemas.

En ese contexto, el interés de esta investigación se orientó a formular un modelo de estrategias de comprensión lectora para el pensamiento crítico y creativo en estudiantes de la institución educativa $\mathrm{N}^{\circ} 16093-J a e ́ n$. La pregunta de indagación queda redactada de la siguiente manera: ¿Cómo el modelo de estrategias de comprensión lectora promueve 
el desarrollo del pensamiento crítico y creativo en los alumnos del segundo grado de educación primaria de la institución educativa N 16093 “José Gálvez” Jaén, 2021?

El estudio se justifica en el aspecto teórico porque se fundamenta en las teorías y competencias cuyos aportes extienden los saberes del investigador y notable de la indagación. En el aspecto metodológico, se trata de una exploración básica con alcance propositivo porque se diseña una propuesta de solución a un tema identificado en el juicio formativo del educando, en el aspecto comunitario su trascendencia radica en que sus aportes van a condescender de soporte para posteriores investigaciones, específicamente, para los docentes que puedan emplear esta estrategia del mismo modo se favorecerá la comunidad educativa.

También, se planteó como objetivo general: Proponer un modelo de estrategias de comprensión lectora para el desarrollo de pensamiento crítico y creativo de escolares de segundo grado de educación primaria de la institución educativa 16093, Jaén, 2021, y como objetivos específicos son: Identificar el nivel de desarrollo del pensamiento crítico y creativo de los estudiantes de segundo grado de educación primaria de la institución educativa 16093; Diseñar el modelo de estrategias de comprensión lectora para desarrollar el pensamiento crítico y creativo de los escolares que participan de la investigación; Validar el modelo de estrategias de comprensión lectora para el desarrollo del pensamiento crítico y creativo, mediante juicio de expertos.

Como hipótesis se planteó que, el modelo de estrategias de comprensión lectora es una herramienta efectiva para promover el desarrollo del pensamiento crítico y creativo en los estudiantes de segundo grado de educación primaria de la Institución Educativa No 16093 “José Gálvez” - Jaén.

\section{METODOLOGÍA}

\subsection{Tipo y diseño de investigación}

La investigación realizada fue básica, de alcance descriptivo propositiva ya que, frente a un problema detectado se propone una solución determinada (Hernández, Fernández y Mendoza, 2018)

En la presente investigación se elaboró un modelo de estrategias de comprensión lectora para desarrollar el pensamiento crítico y creativo de los estudiantes de segundo grado de educación primaria de la Institución Educativa No 16093 José Gálvez de Jaén. 
Desde una perspectiva de método, el método mixto "implica la recopilación y el análisis de datos tanto cuantitativos como cualitativos" (Gallardo, 2017, p.26).

\section{Diseño}

El diseño fue no experimental, para Ñaupas-Paitán et al. (2014) el diseño de investigación es el plan, la estructura, la estrategia general, de carácter flexible, que orienta y guía al investigador para responder al problema planteado. El diseño que se utilizó en esta investigación fue no experimental y de creación propia de la autora, cuyo diagrama es:

Figura 1

\section{Diseño de Investigación}

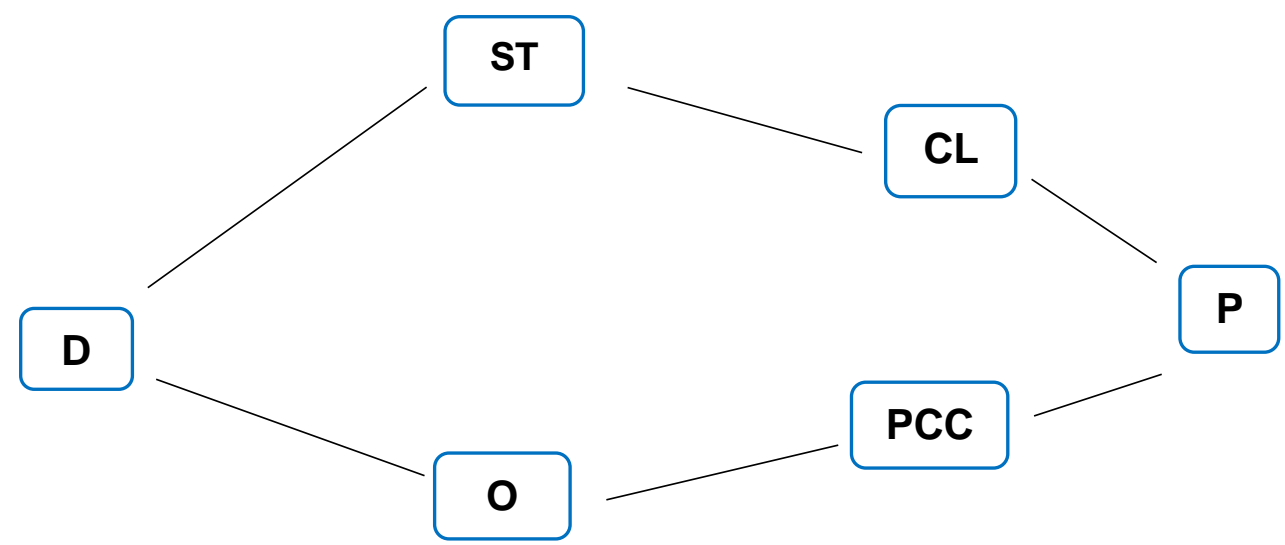

Nota: se presenta la secuencialidad de operaciones. Fuente: elaboración propia.

\section{Donde:}

D: diagnóstico

ST: sustento teórico

O: objetivos

CL: comprensión lectora

PCC: pensamiento crítico y creativo

P: propuesta

\subsection{Variables y operacionalización}

Variable 1 y/o : Modelo de estrategias de comprensión lectora

Variable 2 y/o : Pensamiento crítico y creativo

\subsection{Población, muestra y muestreo}

\section{Población}

La población entendida como la totalidad de sujetos objeto de investigación y que presentan características similares, factible de medir, la conformaron 20 estudiantes de 
segundo grado de educación primaria de la Institución Educativa N 16093 “José Gálvez”, Jaén. En cuanto a la muestra, fue censal o poblacional ya que se tomó en cuenta a la misma población; en tal razón, no se realizó ningún tipo de muestreo porque se trabajó con el 100\% de la población (Toledo, 2016).

\subsection{Técnicas e instrumentos de recolección de datos}

Arias (2006) refiere que "las técnicas de recolección de datos son las distintas formas de obtener información" y los instrumentos son medios y materiales que se emplean para recoger y almacenar datos.

La técnica de observación fue aplicada a través de una guía de observación (Anexo 3 ) que permitió identificar el nivel de desarrollo del pensamiento crítico y creativo, está compuesto por 20 ítems que explican las dos dimensiones; el pensamiento crítico compuesto por los ítems del 1 al 14 y el pensamiento creativo compuesto por los ítems del 15 al 20. Según (Hernández, Fernández y Baptista, 2014) "la observación consiste en el registro sistemático, cálido y confiable de comportamientos o conductas manifiestas" (p. 309).

Se llevó a cabo con una guía de entrevista que fue aplicada al director de la Institución Educativa involucrada en el estudio, consta de 06 preguntas relacionadas al desarrollo del pensamiento crítico y creativo de los estudiantes. Tal como lo indica (Hernández, Fernández y Baptista, 2014) la entrevista es “una conversación que se propone con un fin determinado distinto al simple hecho de conversar".

Se realizó aplicando un cuestionario a los docentes con el fin de recolectar información sobre el pensamiento crítico y creativo de los estudiantes (Anexo 1). Según Palella y Martins (2017) la encuesta es una técnica destinada a obtener datos de varias personas cuyas opiniones interesan al investigador, como instrumento se utilizó el cuestionario. Un cuestionario consiste en un conjunto de preguntas respecto de una o más variables a medir, según Hernández, Fernández y Baptista (2014).

\section{RESULTADOS}

Los resultados de la investigación se plasman según los objetivos planteados. Objetivo específico 1: Identificar el nivel de desarrollo del pensamiento crítico y creativo de los estudiantes de segundo grado de educación primaria de la Institución Educativa $\mathrm{N}^{\circ}$ 16093. 
Análisis del instrumento según los niveles de la dimensión pensamiento crítico y sus correspondientes indicadores:

\section{Tabla 1}

Desarrollo del pensamiento crítico a nivel del indicador Análisis

\begin{tabular}{c|l|c|c}
\hline Indicador & Niveles & Frecuencia & Porcentaje \\
\hline \multirow{3}{*}{ Análisis } & Deficiente & 11 & $55 \%$ \\
& Regular & 8 & $40 \%$ \\
& Bueno & 1 & $5 \%$ \\
& Total & $\mathbf{2 0}$ & $\mathbf{1 0 0 \%}$ \\
\hline
\end{tabular}

Fuente: Guía de observación para evaluar el pensamiento crítico y creativo, dirigido a los estudiantes de segundo grado de educación primaria de la I.E. N. ${ }^{o} 16093$, "José Gálvez"-Jaén.

\section{Figura 1}

Desarrollo del pensamiento crítico a nivel del indicador Análisis

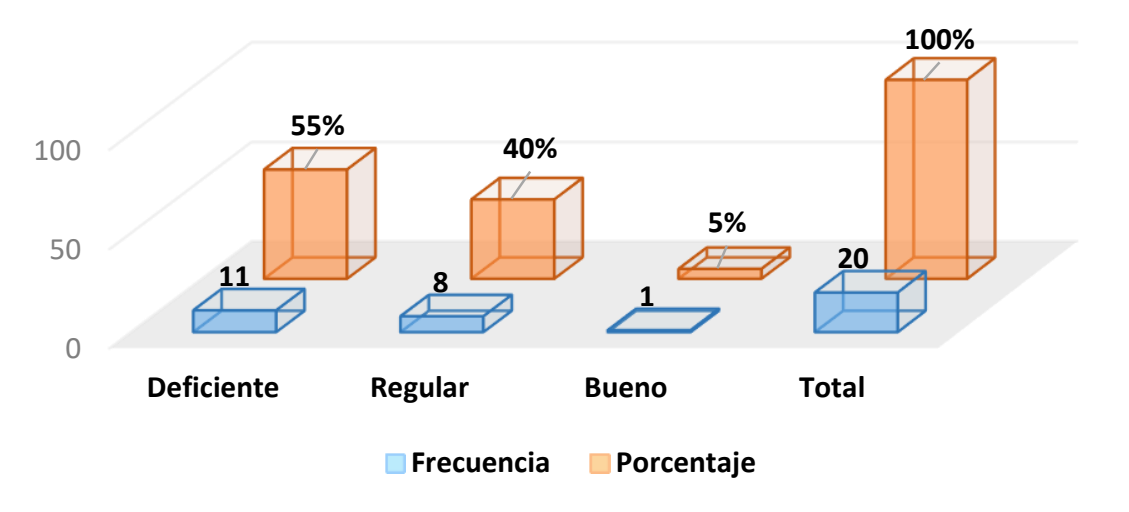

En tabla 1 se puede observar que los estudiantes del segundo grado de educación primaria de la institución educativa N. ${ }^{\circ}$ 16093, "José Gálvez" - Jaén, en el indicador Análisis del pensamiento crítico presentan un 55\% en el nivel deficiente, el $40 \%$ en el nivel regular y el $5 \%$ en el nivel bueno.

\section{Tabla 2}

Desarrollo del pensamiento crítico a nivel del indicador interpretación

\begin{tabular}{rlcc}
\hline \multicolumn{1}{c}{ Indicador } & \multicolumn{1}{c}{ Niveles } & Frecuencia & Porcentaje \\
\hline \multirow{3}{*}{ Interpretación } & Deficiente & 11 & $55 \%$ \\
& Regular & 8 & $40 \%$ \\
& Bueno & 1 & $5 \%$ \\
Total & & $\mathbf{2 0}$ & $\mathbf{1 0 0 \%}$ \\
\hline
\end{tabular}

Fuente: Guía de observación para evaluar el pensamiento crítico y creativo, dirigido a los estudiantes de segundo grado de educación primaria de la I.E. N. ${ }^{\circ}$ 16093, "José Gálvez" - Jaén. 


\section{Figura 2}

Desarrollo del pensamiento crítico a nivel del indicador interpretación

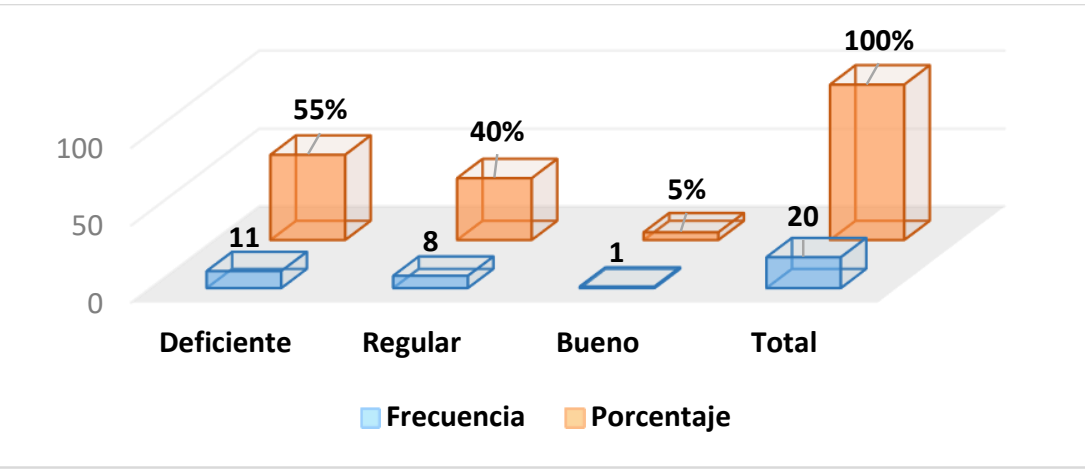

En la tabla 2 se observa que los niños y niñas de segundo grado de primaria de la Institución Educativa No 16093, "José Gálvez" - Jaén, en un 55\% muestran un nivel deficiente en el desarrollo del pensamiento crítico en su indicador interpretación, $40 \%$ en un nivel regular y el $5 \%$ en el nivel bueno.

\section{Tabla 3}

Desarrollo del pensamiento crítico a nivel del indicador Explicación

\begin{tabular}{llcc}
\hline Indicador & Niveles & Frecuencia & Porcentaje \\
\hline \multirow{3}{*}{ Explicación } & Deficiente & 16 & $80 \%$ \\
& Regular & 3 & $15 \%$ \\
Total & Bueno & 1 & $5 \%$ \\
\hline
\end{tabular}

Fuente: Guía de observación para evaluar el pensamiento crítico y creativo, dirigido a los estudiantes de segundo grado de educación primaria de la I.E. N. ${ }^{\circ}$ 16093, "José Gálvez" - Jaén.

\section{Figura 3}

Desarrollo del pensamiento crítico a nivel del indicador Explicación

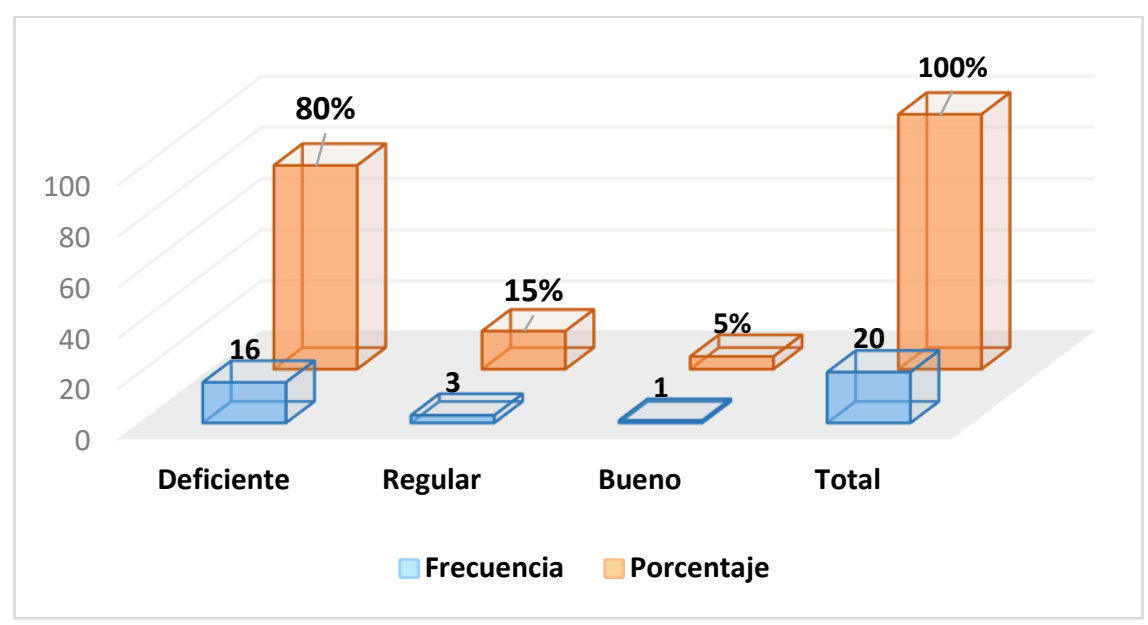


En tabla 3 se puede observar que los estudiantes del segundo grado de educación primaria de la institución educativa N. ' 16093, “José Gálvez” - Jaén, en el indicador Explicación del pensamiento crítico presentan un $80 \%$ en el nivel deficiente, el $15 \%$ en el nivel regular y el $5 \%$ en el nivel bueno.

\section{Tabla 4}

Desarrollo del pensamiento crítico a nivel del indicador Evaluación

\begin{tabular}{llcc}
\hline \multicolumn{1}{c}{ Indicador } & \multicolumn{1}{c}{ Niveles } & Frecuencia & Porcentaje \\
\hline \multirow{3}{*}{ Evaluación } & Deficiente & 15 & $75 \%$ \\
& Regular & 5 & $25 \%$ \\
& Bueno & 0 & $0 \%$ \\
Total & & $\mathbf{2 0}$ & $\mathbf{1 0 0 \%}$ \\
\hline
\end{tabular}

Fuente: Guía de observación para evaluar el pensamiento crítico y creativo, dirigido a los estudiantes de segundo grado de educación primaria de la I.E. N. ${ }^{\circ} 16093$, "José Gálvez"-Jaén.

\section{Figura 3}

Desarrollo del pensamiento crítico a nivel del indicador Evaluación

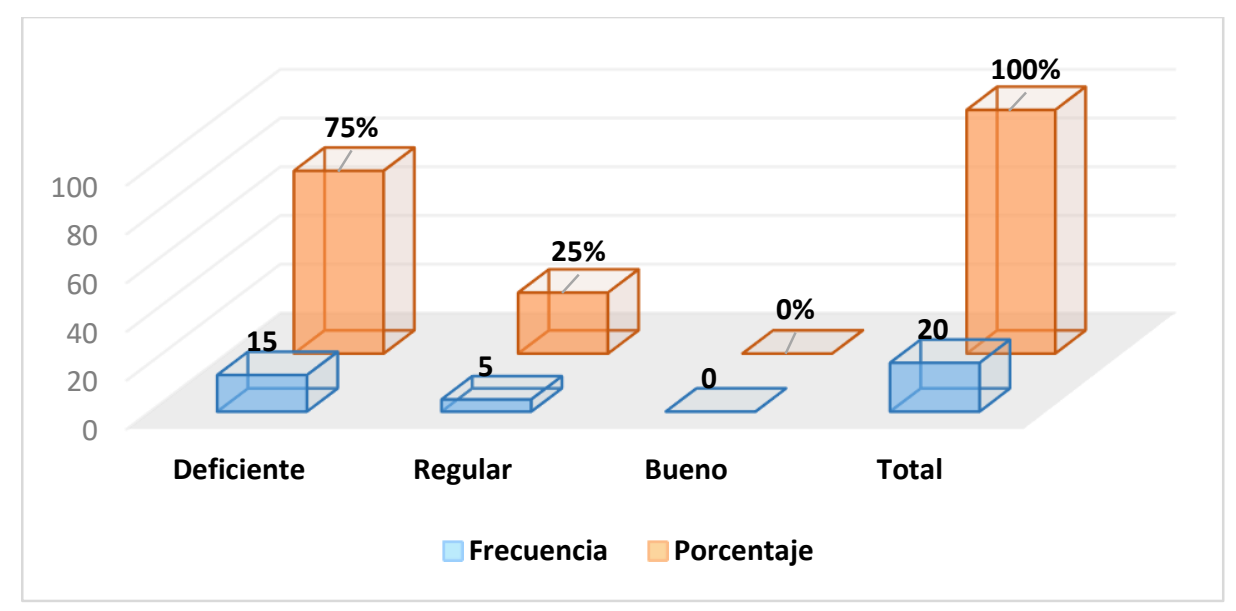

En tabla 4 se puede observar que los estudiantes del segundo grado de educación primaria de la institución educativa N. ${ }^{0}$ 16093, "José Gálvez" - Jaén, en el indicador Evaluación del pensamiento crítico presentan un $75 \%$ en el nivel deficiente, el $25 \%$ en el nivel regular y el $0 \%$ en el nivel bueno. 


\section{Tabla 5}

Desarrollo del pensamiento crítico a nivel de sus cuatro indicadores

\begin{tabular}{lcccc}
\hline \multicolumn{1}{c}{ Niveles } & Análisis & Interpretación & Explicación & Evaluación \\
\hline Deficiente & $55 \%$ & $55 \%$ & $80 \%$ & $75 \%$ \\
Regular & $40 \%$ & $40 \%$ & $15 \%$ & $25 \%$ \\
Bueno & $5 \%$ & $5 \%$ & $5 \%$ & $0 \%$ \\
Total & $100 \%$ & $100 \%$ & $100 \%$ & $100 \%$ \\
\hline
\end{tabular}

Fuente: Guía de observación para evaluar el pensamiento crítico y creativo, dirigido a los estudiantes de segundo grado de educación primaria de la I.E. N. ${ }^{\circ}$ 16093, "José Gálvez"-Jaén.

\section{Figura 5}

Desarrollo del pensamiento crítico a nivel de sus cuatro indicadores

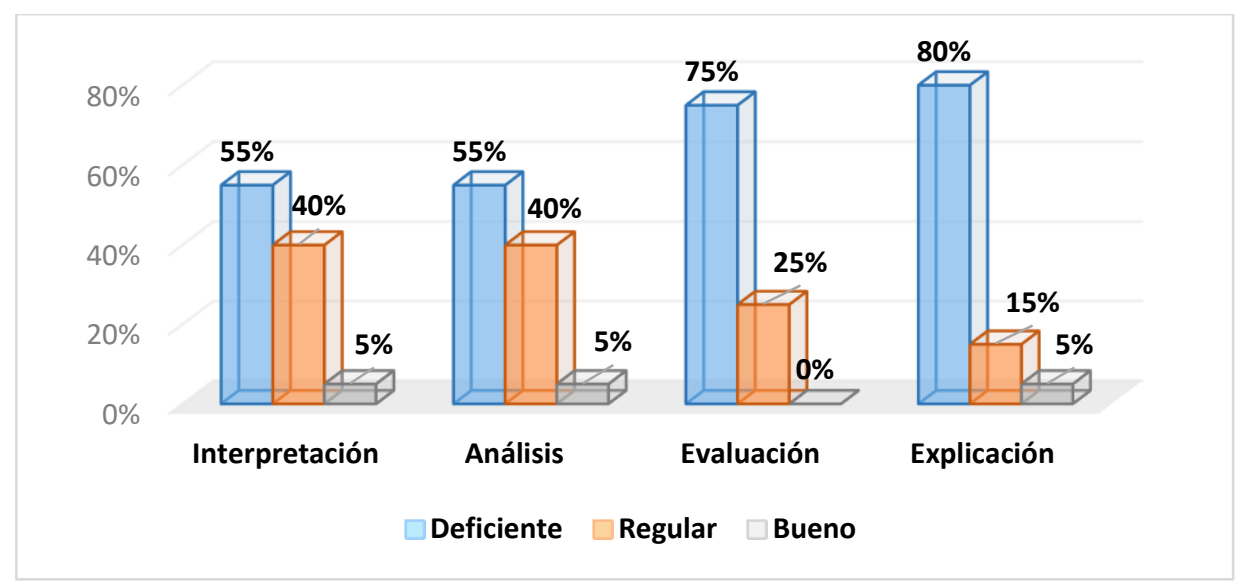

En la tabla 9 se comprueba que los estudiantes del segundo grado de educación primaria de la institución educativa N 16093, “José Gálvez" - Jaén, no han desarrollado aún el pensamiento crítico ya que el porcentaje más alto que alcanzaron fue en el nivel deficiente en todas sus dimensiones.

\section{Tabla 6}

Desarrollo del pensamiento creativo a nivel del indicador Originalidad

\begin{tabular}{lccc}
\hline \multicolumn{1}{c}{ Indicador } & Niveles & Frecuencia & Porcentaje \\
\hline \multirow{3}{*}{ Originalidad } & Deficiente & 13 & $65 \%$ \\
& Regular & 6 & $30 \%$ \\
Total & Bueno & 1 & $5 \%$ \\
\hline
\end{tabular}

Fuente: Guía de observación para evaluar el pensamiento crítico y creativo, dirigido a los estudiantes de segundo grado de educación primaria de la I.E. N. ${ }^{\circ}$ 16093, "José Gálvez" - Jaén. 


\section{Figura 6}

Desarrollo del pensamiento creativo a nivel del indicador Originalidad

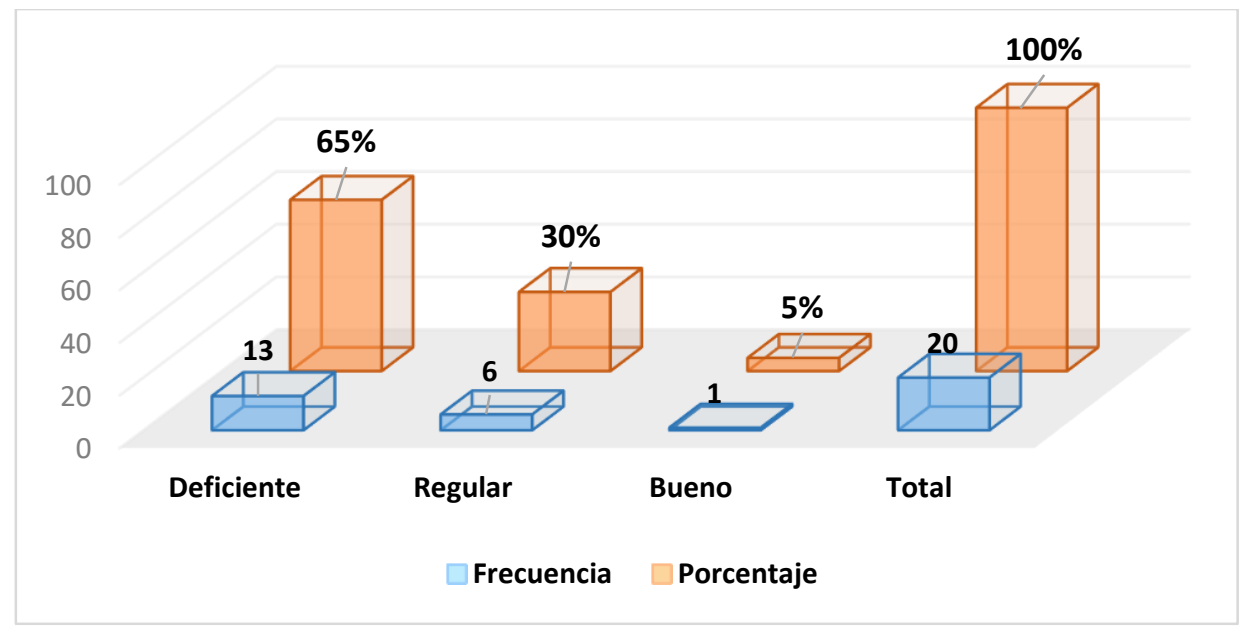

En tabla 6 se puede observar que los estudiantes del segundo grado de educación primaria de la institución educativa N. ${ }^{\circ} 16093$, “José Gálvez" - Jaén, en el indicador Originalidad del pensamiento creativo presentan un $65 \%$ en el nivel deficiente, el $30 \%$ en el nivel regular y el $5 \%$ en el nivel bueno.

\section{Tabla 7}

Desarrollo del pensamiento creativo a nivel del indicador Flexibilidad

\begin{tabular}{clcc}
\hline \multicolumn{1}{c}{ Indicador } & \multicolumn{1}{c}{ Niveles } & Frecuencia & Porcentaje \\
\hline \multirow{3}{*}{ Flexibilidad } & Deficiente & 12 & $60 \%$ \\
& Regular & 6 & $30 \%$ \\
& Bueno & 2 & $10 \%$ \\
Total & & $\mathbf{2 0}$ & $\mathbf{1 0 0 \%}$ \\
\hline
\end{tabular}

Fuente: Guía de observación para evaluar el pensamiento crítico y creativo, dirigido a los estudiantes de segundo grado de educación primaria de la I.E. N. ${ }^{\circ}$ 16093, "José Gálvez" - Jaén.

\section{Figura 7}

Desarrollo del pensamiento creativo a nivel del indicador flexibilidad

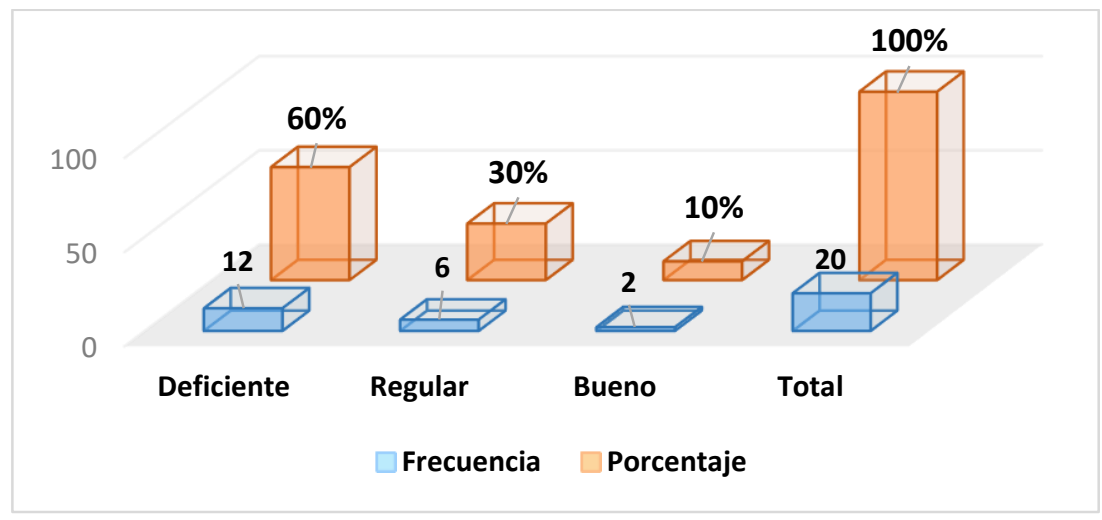


En tabla 7 se puede observar que los estudiantes del segundo grado de educación primaria de la institución educativa N. ${ }^{\circ}$ 16093, "José Gálvez" - Jaén, en el indicador flexibilidad del pensamiento creativo presentan un $60 \%$ en el nivel deficiente, el $30 \%$ en el nivel regular y el $10 \%$ en el nivel bueno.

\section{Tabla 8}

Desarrollo del pensamiento creativo a nivel del indicador Fluidez.

\begin{tabular}{llcc}
\hline Indicador & Niveles & Frecuencia & Porcentaje \\
\hline \multirow{3}{*}{ Fluidez } & Deficiente & 8 & $40 \%$ \\
& Regular & 6 & $30 \%$ \\
Total & Bueno & 6 & $30 \%$ \\
\hline
\end{tabular}

Fuente: Guía de observación para evaluar el pensamiento crítico y creativo, dirigido a los estudiantes de segundo grado de educación primaria de la I.E. N. ${ }^{\circ} 16093$, "José Gálvez"-Jaén.

\section{Figura 8}

Desarrollo del pensamiento creativo a nivel del indicador fluidez

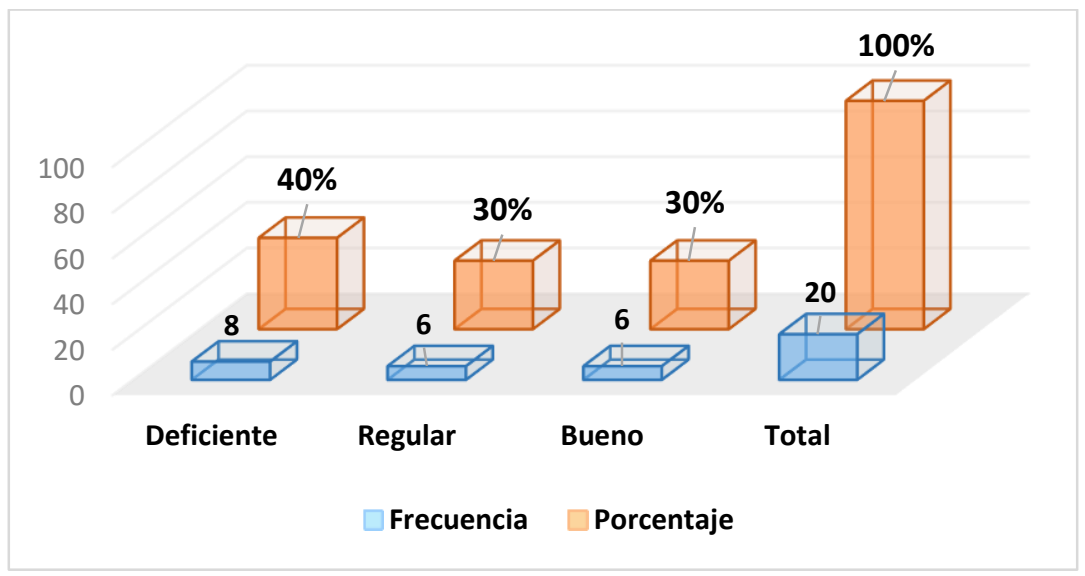

En tabla 8 se puede observar que los estudiantes del segundo grado de educación primaria de la institución educativa N. ${ }^{\circ}$ 16093, “José Gálvez" - Jaén, en el indicador fluidez del pensamiento creativo presentan un $40 \%$ en el nivel deficiente, el $30 \%$ en el nivel regular y el $30 \%$ en el nivel bueno. 


\section{Tabla 9}

Desarrollo del pensamiento creativo a nivel del indicador Elaboración

\begin{tabular}{llcc}
\hline Indicador & Niveles & Frecuencia & Porcentaje \\
\hline \multirow{3}{*}{ Elaboración } & Deficiente & 12 & $60 \%$ \\
& Regular & 5 & $25 \%$ \\
Total & Bueno & 3 & $15 \%$ \\
\hline
\end{tabular}

Fuente: Guía de observación para evaluar el pensamiento crítico y creativo, dirigido a los estudiantes de segundo grado de educación primaria de la I.E. N. ${ }^{\circ}$ 16093, "José Gálvez"-Jaén.

\section{Figura 9}

Desarrollo del pensamiento creativo a nivel del indicador elaboración

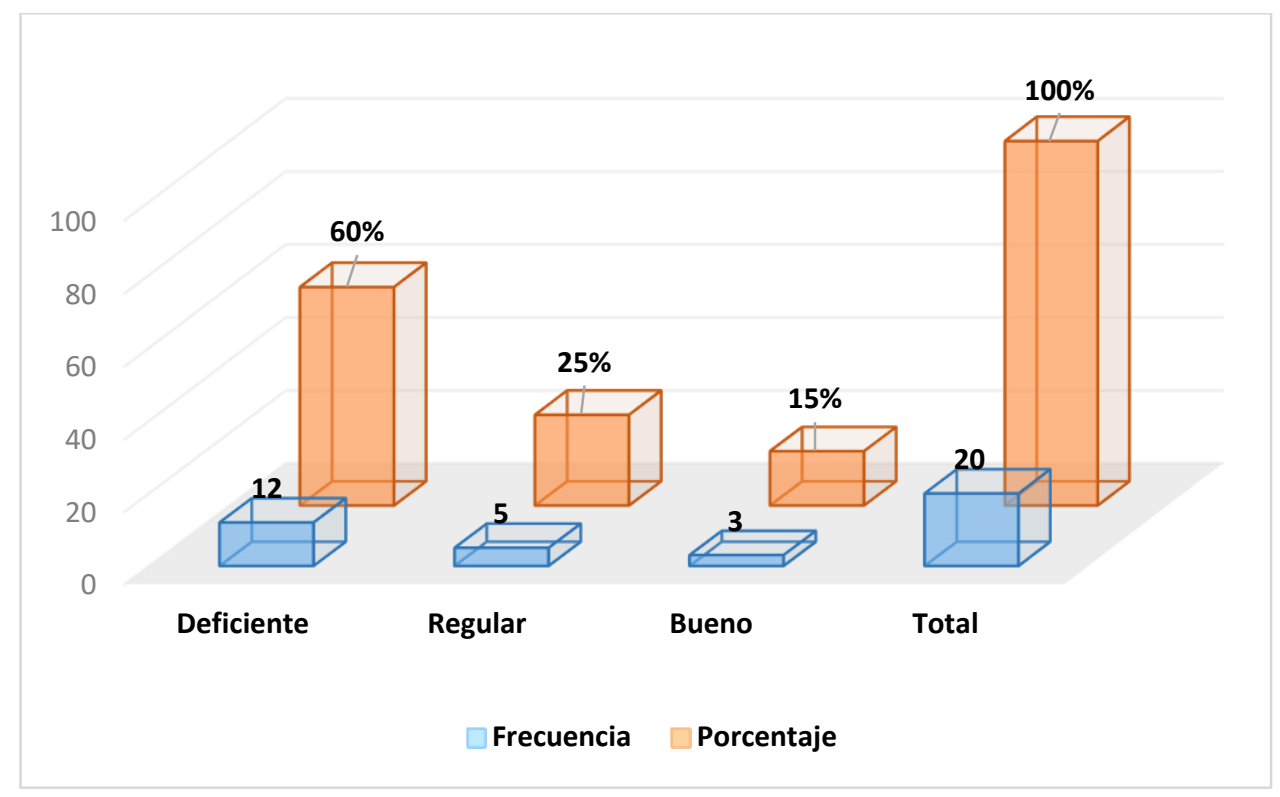

En tabla 9 se puede observar que los estudiantes del segundo grado de educación primaria de la institución educativa N. ${ }^{\circ} 16093$, “José Gálvez" - Jaén, en el indicador Elaboración del pensamiento creativo presentan un $60 \%$ en el nivel deficiente, el $25 \%$ en el nivel regular y el $15 \%$ en el nivel bueno. 


\section{Tabla 10}

Desarrollo del pensamiento creativo a nivel de sus cuatro indicadores

\begin{tabular}{lcccc}
\hline \multicolumn{1}{c}{ Niveles } & Originalidad & Flexibilidad & Fluidez & Elaboración \\
\hline Deficiente & $65 \%$ & $60 \%$ & $40 \%$ & $60 \%$ \\
Regular & $30 \%$ & $30 \%$ & $30 \%$ & $25 \%$ \\
Bueno & $5 \%$ & $10 \%$ & $30 \%$ & $15 \%$ \\
Total & $\mathbf{1 0 0 \%}$ & $\mathbf{1 0 0 \%}$ & $\mathbf{1 0 0 \%}$ & $\mathbf{1 0 0 \%}$ \\
\hline
\end{tabular}

Fuente: Guía de observación para evaluar el pensamiento crítico y creativo, dirigido a los estudiantes de segundo grado de educación primaria de la I.E. N. ${ }^{\circ} 16093$, "José Gálvez"-Jaén.

\section{Figura 10}

Desarrollo del pensamiento creativo a nivel de sus cuatro indicadores

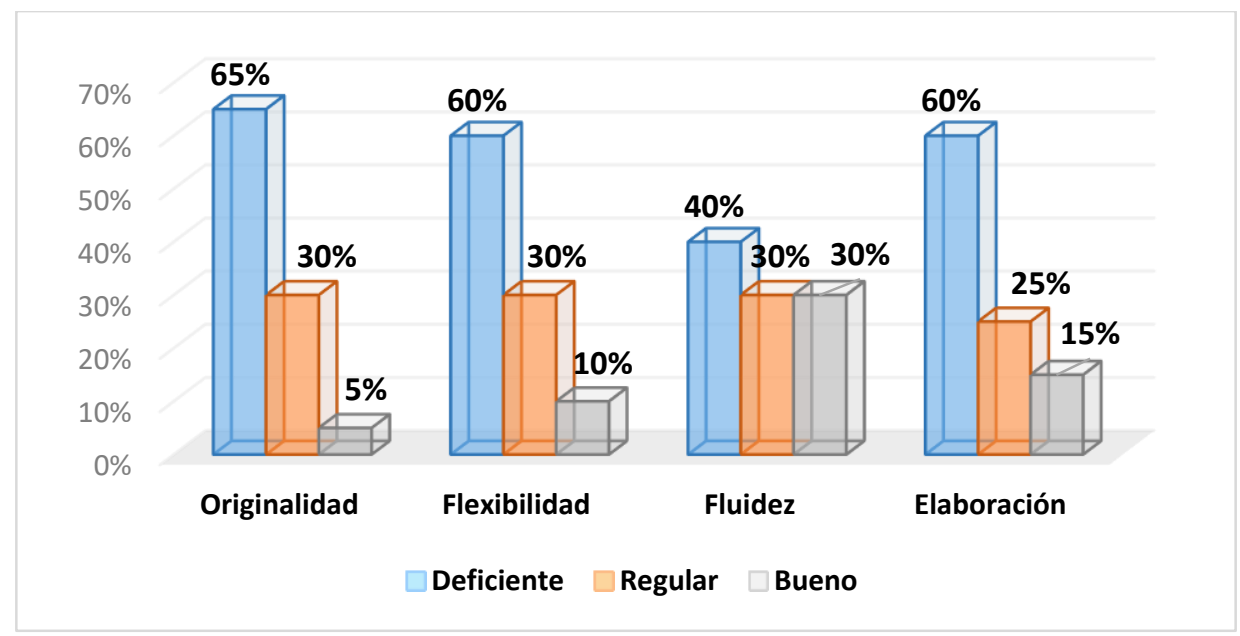

En la tabla 10 se comprueba que los estudiantes del segundo grado de educación primaria de la institución educativa N ${ }^{\circ} 16093$, “José Gálvez" - Jaén, no han desarrollado aún el pensamiento creativo ya que el porcentaje más alto que alcanzaron fue en el nivel deficiente en todas sus dimensiones.

\section{DISCUSIÓN}

Se desarrolló una investigación con alcance descriptivo donde se tuvo como objeto de estudio y variable central al pensamiento crítico y creativo, categoría compuesta por dos formas de pensamiento, de un lado, la criticidad y, de otro, la creatividad. En lo concerniente al pensamiento crítico, según Pérez, Hernández y Alcántara (2017) pensar con sentido crítico es razonar o meditar con mente abierta identificando y valorando, cuando resulte necesario, sus supuestos, implicaciones y derivaciones prácticas; además, 
interactuar y establecer comunicación efectiva con las personas de su entorno, buscando soluciones a los problemas complicados; siguiendo a estos autores, el pensamiento creativo, lo definen como una capacidad que no está anclada a lo intelectual sino que se exterioriza en la interacción de los concepciones de una persona con el entorno donde se llevan a cabo.

Entonces, en base a estas premisas, se ejecutó una investigación con enfoque cuantitativo, evaluándose el estado de la variable dependiente en los estudiantes de la muestra, buscando alcanzar el objetivo general orientado a proponer un modelo de estrategias de comprensión lectora para el desarrollo de pensamiento crítico y creativo. Lograr este objetivo implicó a su vez, alcanzar los objetivos específicos, siendo el primero de ellos, identificar el nivel de desarrollo del pensamiento crítico y creativo de los estudiantes del grupo muestral, esta variable se desagregó en dos dimensiones: pensamiento crítico y pensamiento creativo.

Después del procesamiento de los datos recogidos, los resultados indican que el $55 \%$ de estudiantes se ubicaron en el nivel deficiente en el desarrollo del pensamiento crítico, según el indicador análisis; al examinar este dato, se puede inferir que la mayoría de estudiantes de la muestra requiere apoyo pedagógico a fin de desarrollar su capacidad para examinar adecuadamente la información que recoge o accede, atender con cuidado las opiniones que emiten las personas de su entorno, exponer soluciones posibles y equiparar sus pros y contras, con la finalidad de descubrir la opción mejor y, de este modo, adoptar una adecuada decisión a fin de solucionar algún problema o situación al que se está enfrentando, como lo comenta Hall (2019). Asimismo, en el indicador interpretación, de modo coincidente, se halló que el 55\% también poseen nivel deficiente en el desarrollo de este indicador del pensamiento crítico, infiriéndose que a la mayoría de estudiantes hace falta desarrollar su capacidad para manifestar o exponer el sentido que posee el contenido, principalmente el de un texto, dicho de otro modo, saber interpretar significa que la persona es capaz para resumir las ideas de un texto leído, además, enfocarlas en un contexto más amplio.

También se recolectó datos sobre el indicador explicación del pensamiento crítico, los estudiantes investigados en un $80 \%$ presentan nivel deficiente, esto indica que la gran mayoría de ellos tienen capacidad para exponer un tema o asunto, una opinión o un texto haciéndolo de forma clara y precisa a fin de que se torne más inteligible, con la 
explicación es posible sacar a la luz o concebir más visiblemente el sentido o contenido de algo. De igual manera, en el indicador Evaluación del pensamiento crítico el $75 \%$ de estudiantes se ubicó en el nivel deficiente, dato que conduce a afirmar que un porcentaje significativo de ellos requiere más ejercicio de su capacidad para valorar el contenido de un texto, el actuar de sus personajes y emitir sus apreciaciones sobre la información contenida en un texto; como lo precisa la Universidad Católica de Oriente (2017) La evaluación en cuanto sea valoración, cuestionamiento, averiguación, análisis, constituye algo natural, inherente y normal al quehacer humano. No constituye asunto o aspecto "externo o superpuesto", más bien es algo que corresponde a su propia naturaleza y necesidad.

En base a las descripciones y análisis de los hallazgos en cuanto a la dimensión pensamiento crítico, se puede confirmar lo señalado por Lev Vigotsky en su teoría sociocultural formulada hacia 1979, donde el autor plantea que el desarrollo personal no puede entenderse sin el contexto social y cultural en el que la persona se encuentra inmersa, en esa perspectiva, según el autor, los procesos cognitivos superiores del individuo como el pensamiento crítico, la toma de decisiones, entre otros, tienen su origen y están conectados con los procesos sociales. De igual manera, se debe respaldar los resultados alcanzados con los aportes teóricos de Jean Piaget en su teoría del desarrollo cognitivo propuesta en el año 1986, según el estudioso cuando el estudiante piensa críticamente hace uso consciente y sistemático de sus recursos intelectuales de tal forma que es capaz de comprender, explicar, gestionar, decidir o crear algo, en esa perspectiva, su pensamiento crítico es un pensamiento instrumental por excelencia y le capacita para resolver adecuadamente problemas o situaciones problemáticas, además, adoptar decisiones eficientes y efectivas, de allí su trascendencia en el proceso de aprendizaje, surgiendo la necesidad de estimular desde temprana edad el pensamiento crítico.

Los resultados obtenidos también conducen a señalar que, si la mayoría de estudiantes está en nivel deficiente de su pensamiento crítico, significa que este proceso intelectual decisivo, intencionado y autocontrolado no está cumpliendo con el objetivo de construir un juicio razonable, poniendo en ejercicio sus facultades intelectuales y activar su capacidad para analizar, interpretar, explicar y evaluar la información procedente del mundo exterior; además, que su juicio razonable se torne en explícito y justificado en 
función a los conceptos que se posee de su contexto fundamentados en criterios convenientes.

\section{CONCLUSIONES}

El pensamiento crítico y creativo en la mayoría de estudiantes de segundo grado de educación primaria de la Institución Educativa 16093, no está desarrollado ya que tanto para el pensamiento crítico como para el pensamiento creativo predominó el nivel deficiente, en tal razón, se requiere intervención pedagógica con estrategias que permitan revertir la dificultad encontrada.

Una de las herramientas eficaces para desarrollar el pensamiento crítico y creativo lo constituye la lectura comprensiva, por ello, se diseñó un modelo de estrategias de comprensión lectora para desarrollar el pensamiento crítico y creativo de los escolares investigados; el mismo que se basó en los aportes de la Teoría del aprendizaje significativo de David Ausubel y los alcances pedagógicos de Isabel Solé.

A fin de asegurar la viabilidad y posterior efectividad del modelo de estrategias de comprensión lectora propuesto, fue objeto de revisión y validación a juicio de expertos, quienes finalmente emitieron su conformidad respecto a que la propuesta contribuirá con el desarrollo del pensamiento crítico y creativo de los estudiantes. 


\section{DISEÑO GRÁFICO DE LA PROPUESTA}
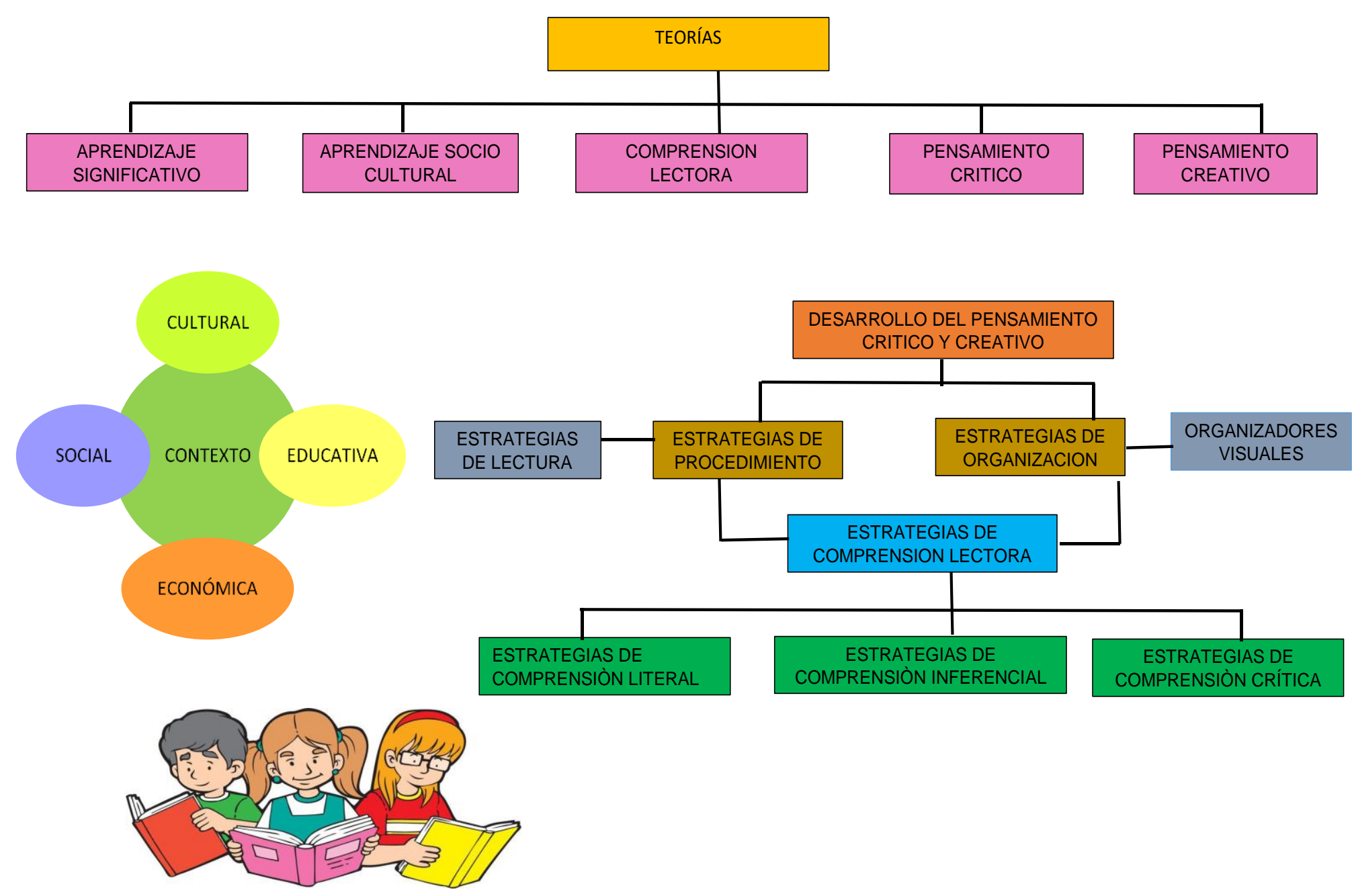

Ciencia Latina Revista Científica Multidisciplinar, Ciudad de México, México.

ISN 2707-2207 / ISSN 2707-2215 (en línea), enero-febrero, 2022, Volumen 6, Número 1. https://doi.org/10.37811/cl_rcm.v6i1.1493 p199 


\section{REFERENCIAS BIBLIOGRAFICAS}

Arias. F. (2006) El proyecto de investigación: Introducción a la investigación científica

(5ta ed.). Caracas, Venezuela: Editorial Episteme, C.A. https://www.eumed.net/tesis-doctorales/2012/zll/metodologia-investigacion.html

Bretel, L. (2017). Diálogos educativos/Entrevistado por Lilia Calmet. Revista Fundación Wiese.

https://www.fundacionwiese.org/blog/wpcontent/uploads/2018/06/Entrevista-aLuis-Bretel.pdf.

Gallardo, Echenique, E. E. (2017). Metodología de la Investigación: manual autoformativo interactivo https://repositorio.continental.edu.pe/bitstream/20.500.12394/4278/1/DO_UC_E G_MAI_UC0584_2018.pdf

Hernández-Sampieri, R., Fernández-Collado, C., \& Baptista-Lucio, M. del P. (2014). Metodología de la investigación (6ta ed.). México D.F.: McGraw-Hill / Interamericana Editores, S.A. DE C.V

Hernández, R.; Fernández, C.; Baptista, P. (2014). Metodología de la Investigación. México: Mc Graw Hill Educatión, 6ta. Edición, p. 200.

Hernández-Sampieri, R. \& Mendoza, C (2018). Metodología de la investigación. Las rutas cuantitativa, cualitativa y mixta, Ciudad de México, México: Editorial Mc Graw Hill Education, Año de edición: 2018, ISBN: 978-1-4562-6096-5, 714 p

Meneses, N. (22 de enero de 2020). Para fomentar el pensamiento crítico, garanticemos que todos salen de la ESO sabiendo leer y escribir. El País. https://elpais.com/economia/2020/01/22/actualidad/1579697337_265428.html

Ministerio de Educación. (2017). Perú, ¿Cómo vamos en educación? Primera edición, Lima. http://escale.minedu.gob.pe/c/document_library/get_file?uuid=2e13b696a8f6-4206-9276-5db05a8b4702\&groupId=10156

Ñaupas-Paitán, H., Mejía-Mejía, E., Novoa-Ramírez, E., \& Villagomez-Páucar, A. (2014). Metodología de la investigación cuantitativa-cualitativa y redacción de la tesis (4th ed.). Bogotá, Colombia: Ediciones de la U.

OCDE. (2019). Estrategia de competencias de la OCDE 2019. competencias para construir un futuro mejor. In Fundación Santillana, 2019. para la edición en español. https://doi.org/10.1787/e3527cfb-es.pdf 
Palella, M. (2017: 122). Metodología de la investigación. https://metinvest.jimdofree.com/t\%C3\%A9cnicas/

Pérez, I., Hernández, J. y Alcántara, E. A. (2017). Pensamiento crítico y creativo en estudiantes de educación superior: un análisis desde la teoría de la actividad. https://www.comie.org.mx/congreso/memoriaelectronica/v14/doc/0705.pdf

Piaget, J. (1994). El nacimiento de la inteligencia del niño. México: Editorial Grijalbo

Proyecto Educativo Regional. (2017). Balance y recomendaciones.

https://www.cne.gob.pe/uploads/publicaciones/publicacion/2018/balance-pen2017-2018.pdf

Rodríguez, A. (2019). Pensamiento Crítico: Características, Habilidades y Actitudes. https://www.lifeder.com/ pensamiento-crítico/

Solé, I. (2007). La lectura como transformación del pensamiento. Proyecto de Lectura para Centros Escolares (plec). Madrid: Fundación Germán Sánchez Ruipérez

Toledo, D, De León, N. (2016) Población y muestra http://hdl.handle.net/20.500.11799/63099

Torrent, L. (2016). Pensamiento crítico y creatividad https://www.lampadia.com/analisis/educacion/pensamiento-critico-ycreatividad/

http://hdl.handle.net/20.500.11799/63099

UNESCO. (2019). ¿Qué se espera que aprendan los estudiantes de América Latina y el Caribe? Organización de Las Naciones Unidas Para La Educación, La Ciencia y La Cultura y La Oficina Regional de Educación Para América Latina y El Caribe (OREALC/UNESCO Santiago) Organización de Las Naciones Unidas Para La Educación, La Ciencia y La Cultura y La, Erce, 188. https://unesdoc.unesco.org/ark:/48223/pf0000373982

Vygotsky, L. S. (1979) El desarrollo de los procesos psicológicos superiores. Buenos Aires: Grijalbo. 